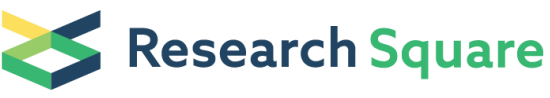 \\ Preprints are preliminary reports that have not undergone peer review. \\ They should not be considered conclusive, used to inform clinical practice, or referenced by the media as validated information.
}

\section{Overexpression of Sesame Polyketide Synthase A Leads to Abnormal Pollen Development in Arabidopsis}

Tianyu Li

Ministry of Agriculture of the People's Republic of China, Chinese Academy of Agricultural Sciences Ting Zhou

Ministry of Agriculture of the People's Republic of China, Chinese Academy of Agricultural Sciences

Yuanxiao Yang

Ministry of Agriculture of the People's Republic of China, Chinese Academy of Agricultural Sciences

Hongyan Liu

Ministry of Agriculture of the People's Republic of China, Chinese Academy of Agricultural Sciences

Fang Zhou

Ministry of Agriculture of the People's Republic of China, Chinese Academy of Agricultural Sciences

\section{Senouwa Segla Koffi Dossou}

Ministry of Agriculture of the People's Republic of China, Chinese Academy of Agricultural Sciences

Yingzhong Zhao ( $\nabla$ zhaoyz63@163.com )

Ministry of Agriculture of the People's Republic of China, Chinese Academy of Agricultural Sciences

\section{Research Article}

Keywords: Sesame, Male-sterility, Exine, Sporopollenin, SiPKSA

Posted Date: August 13th, 2021

DOl: https://doi.org/10.21203/rs.3.rs-778472/v1

License: (c) (i) This work is licensed under a Creative Commons Attribution 4.0 International License. Read Full License

Version of Record: A version of this preprint was published at BMC Plant Biology on April 2nd, 2022. See the published version at https://doi.org/10.1186/s12870-022-03551-7. 


\section{Abstract}

Background: Sesame is a great reservoir of bioactive constituents and unique antioxidant components and is widely used for its nutritional and medicinal value. The expanding demands for sesame seeds are putting pressure on sesame breeders to develop reliable high-yielding varieties. Heterosis utilization is an efficient way to increase sesame yield. Polyketide synthases (PKSs) are critical enzymes in the biosynthesis of sporopollenin, a primary component of pollen exine. Their in planta functions are being investigated for application in crop breeding.

Results: In this study, we cloned the sesame POLYKETIDE SYNTHASE A (SIPKSA) and examined its function in male sterility. SiPKSA was specifically expressed in sesame flower buds, and its expression was significantly higher in sterile sesame anthers than in fertile anthers at the tetrad and microspore development stage. Further overexpression of SiPKSA in Arabidopsis caused transgenic plants male sterile. Ultrastructural observation showed that the pollen grains of SiPKSA-overexpressing plants contained few cytoplasmic inclusions and exhibited an abnormal pollen wall structure, with a thicker exine layer compared with wild type. In agreement with it, the expression of a set of sporopollenin biosynthesis-related genes and the contents of fatty acids and phenolics were significantly altered in anthers of SiPKSA-overexpressing plants compared with wild type during anther development.

Conclusion: These findings highlighted that overexpression of SiPKSA in Arabidopsis might cause excessive sporopollenin biosynthesis to influence pollen and pollen wall development, leading to male sterile, suggesting that its manipulation might improve hybrid breeding in sesame and other crop species.

\section{Background}

Sesame (Sesamum indicum L.) is one of the most ancient oil crops and is widely used for its high-quality products. Its seeds are a great reservoir of oil, protein, unsaturated fatty acids, lignans, vitamins, and minerals [1, 2]. Many studies revealed that sesame lignans were associated with various healthpromoting effects against lifestyle diseases [3-6]. Accordingly, the sesame seeds market is expanding, giving a big pressure to sesame breeders to develop environment stable and high-yielding varieties. Hybrid breeding strategy based on male sterile lines is one of the most reliable techniques to increase crop yield. Therefore, deciphering the molecular mechanism of fertility-related genes in sesame might provide genetic resources and new ideas for its utilization of heterosis.

Pollen is a critical organ in angiosperms plants reproduction. Male reproductive organ development is a complex biological process that consists of various biosynthesis events, among which pollen wall formation is essential for pollen viability and fertility $[7,8]$. Any defects during the pollen wall formation may lead to abnormal pollen and further male sterility [9]. The pollen wall is an important structure of the outer surface of pollen. It protects microspores against the harm of harsh environmental conditions [10], and is essential for species-specific pollen-stigma recognition [11]. It is principally comprised of three layers: the pollen coat, the outer exine, and the inner intine. The pollen exine patterning mainly includes 
three developmental events: primexine formation, callose wall formation, and sporopollenin synthesis [7, $10,12]$. Sporopollenin, a complex of fatty acids and phenolic compounds, represents the principal component that constitutes the pollen exine architecture [13].

Studies in Arabidopsis revealed that the sporopollenin biosynthesis occurs in tapetal secretory cells after the temporary callose wall surrounding tetrads is degraded, and the primexine is deposited [14]. Other studies in rice, wheat, and barley showed that pollen development in all plant species involves identical key genes, stages and conserves similar regulatory pathways [7,15-18]. Among genes that contribute to vibrant and fertile pollen formation, polyketide synthases are the key ones [15, 16]. The Arabidopsis $p k s a / l a p 6$ or $p k s b / l a p 5$ mutant produces abnormal pollen exine, and the double mutant produced collapsed pollen without exine deposition and was male sterile $[19,20]$. Kim et al. demonstrated that Arabidopsis POLYKETIDE SYNTHASE A/PKSA and PKSB (also known as LAP6 and LAP5, respectively) interacted continuously with other pollen exine biosynthesis genes [19]. The integration of the increasing genomic information and advanced genetic tools is facilitating the transfer of information from Arabidopsis to other plants, including Physcomitrella patens, rice, and rapeseed, in which the orthologues of PKSA have been identified and characterized for application in hybrid breeding [21-23].

In sesame, although early studies reported strong heterosis [24-26], few nuclear male sterile lines have been used in hybrid breeding $[27,28]$. Studies on sesame male sterility were limited to the identification of two genes, Sesamum indicum male sterility 1 (SiMs1) [29] and Sesamum indicum cell wall invertase 1 (Sicwinv1) [30]. In the present study, the sesame polyketide synthase A (SiPKSA) was cloned and functionally characterized to involve in male reproduction. Our results suggested that SiPKSA might be involved in sporopollenin biosynthesis to affect pollen and pollen wall formation.

\section{Results}

\section{Clone and sequence analysis of SiPKSA}

Sesame polyketide synthase, the orthologue of Arabidopsis PKSA, designated SiPKSA (Sesamum indicum Polyketide Synthase A), was cloned using the CDNA of sesame anthers at the tetrad stage as the template. The open reading frame (ORF) of SiPKSA was 1191 bp, encoding 396 amino acids, with an isoelectric point of 6.41 , a fat index of 95.33 , and a molecular weight of $43.6 \mathrm{kDa}$.

Multiple sequence alignment of SiPKSA with other PKSA proteins reported in Arabidopsis (Arabidopsis thaliana), rice (Oryza sativa), and rape (Brassica napus) showed that SiPKSA contained typical chalcone/stilbene synthase N-terminal domain (25-242 bp), C-terminal domain (252-396 bp) and various conserved active sites, such as polypeptide binding site, malonyl-CoA binding site, and product binding site (Fig. 1A). As well, it contained catalytic triad Cys-His-Asn, which is common and important in the PKSs family (Fig. 1A). Phylogenetic analysis further confirmed that SiPKSA clustered together with PKSA proteins in other species (Fig. 1B), confirming that it encoded a type III polyketide synthase A protein. 
In addition, the promoter region of the SiPKSA was analyzed using the PLACE and PLANTCARE databases. It was found that a large number of formal elements were annotated as MYB recognition sites, light-responsive elements, and auxin-responsive elements. Notably, multiple copies of AGAAA, which was considered as an anther- and/or pollen-specific cis-regulatory motif, were predicted in the promoter region of SiPKSA. Other motifs, such as Q-element and GTGA-motif, related to pollen development were also predicted in the promoter region (Table S2). These results indicated that SiPKSA was a type III polyketide synthase A protein and might be involved in pollen development in sesame.

\section{SiPKSA is predominantly expressed in reproductive tissue}

The expression profile of SiPKSA in different tissues was analyzed. It was shown that SiPKSA was especially expressed in flower buds (Fig. 2A and 2B), indicating its function in sesame might be related to plant reproduction. Further, to determine the potential role of SiPKSA in sesame male sterility, we compared the expression pattern of SiPKSA in sterile and fertile sesame anthers at different developmental stages. We observed that the expression of SiPKSA was significantly higher in sterile sesame anthers at the tetrad stage and microspore development stage compared with the fertile anthers (Fig. 2C and 2D), suggesting that SiPKSA might be related to sesame male fertility.

\section{Overexpressing SiPKSA in Arabidopsis caused transgenic plants male sterile}

To further assess the in vivo function of SiPKSA in the anther development, SiPKSA was overexpressed in Arabidopsis. Several T3 generation transgenic plants were harvested, and two homozygous lines, in which SiPKSA expression was highly overexpressed, were selected for phenotypic and functional analysis (Fig. 3A). The expression level of the Arabidopsis homologous gene AtPKSA was also detected. The results showed that there were no significant differences among SiPKSA-overexpressing plants, wild type and plants transformed with empty vector in terms of the expression level of AtPKSA both in seedlings and anthers at different developmental stages (Fig. S1A-C), suggesting that the phenotype of transgenic plants in the present study was caused by SiPKSA.

As shown in Fig. 3B, the siliques of SiPKSA-overexpressing plants were flat and short compared with those of the wild type (WT) and plants transformed with empty vector (EV) (Fig. 3C-E). The acetocarmine staining showed that the pollen grains of WT and EV were plump and darkly stained (Fig. 3F and $3 \mathrm{H}$ ). While, the pollen grains of SiPKSA-overexpressing plants were uncolored and smaller compared with the controls, indicating the pollen grains of SiPKSA-overexpressing plants were poor vigor (Fig. 3G). Scanning electron microscope (SEM) further confirmed that the WT pollen grains were normal and those of the SiPKSA-overexpressing plants were abnormal. The WT pollen grains were full with a typical sculpture surface (Fig. $3 \mathrm{I}$ and $3 \mathrm{~J}$ ). In contrast, the pollen grains of the SiPKSA-overexpressing plants were shrunken 
and collapsed (Fig. 3K and $3 \mathrm{~L}$ ). These results indicated that SiPKSA-overexpressing plants were male sterile.

\section{Abnormal pollen and pollen wall development in SiPKSA- overexpressing Arabidopsis plants}

Transmission electron microscopy (TEM) observation was further performed to investigate the ultrastructure of pollen development. According to the cytological events, Arabidopsis anther development was characterized into 14 stages, and the microspores were differentiated into mature pollen during stages 9-12 [8]. Our results showed that, during stages $9-10$, the tapetum of WT contained small vacuoles and abundant plastids filled with plastoglobuli (Fig. 4A). The microspores of WT were rich in inclusions and embedded with a regular primary structure of the exine (Fig. 4B). However, in the tapetal cells of SiPKSA-overexpressing plants, large cavities and less substance were observed (Fig. 4C), the microspores of SiPKSA-overexpressing plants were obviously abnormal, with few cytoplasmic components, and the sporopollenin particles were abundant accumulated on the microspore surface (Fig. 4D) During stage 11-12, the pollen of WT exhibited a typical normal pollen wall with obvious exine and intine layers (Fig. 4E). In contrast, the pollen grains of SiPKSA-overexpressing plants were seriously collapsed, with irregular exine and intine (Fig. 4F). Further measurement of the thickness of the pollen exine during the stages $9-10$ and 11-12 showed that, compared with the WT, the exine layer of SiPKSAoverexpressing plants was significantly thicker than WT (Fig. 5A-E).

\section{Overexpression of SiPKSA disrupted the expression of sporopollenin biosynthesis-related genes}

To further explore whether sporopollenin biosynthesis was affected in anthers of SiPKSA-overexpressing plants, the expression of several sporopollenin synthesis-related genes was analyzed by qRT-PCR, including AtMS2, AtCYP704B1, AtTKPR1, and AtTKPR2. As shown in Fig. 6, comparing to WT, the expression of AtMS2, AtCYP704B1, AtTKPR1 and AtTKPR2 were up-regulated in anthers of SiPKSAoverexpressing plants during stage $6-8$. During stages $9-10$, there were no significant differences among SiPKSA-overexpressing plants, WT, and EV in terms of the expression of AtMS2, AtCYP704B1, AtTKPR1, except for AtTKPR2, which expression was also up-regulated. These results indicated that the expressions of sporopollenin biosynthesis-related genes were influenced by the high expression of SiPKSA in transgenic Arabidopsis.

\section{Disrupted fatty acids metabolism occurred in anthers of SiPKSA-overexpressing plants}


Pollen exine is mainly composed of sporopollenin, which was a complex biopolymer constituted of fatty acids, phenols, and their derivatives [38]. Therefore, the contents of fatty acids and their derivates were detected by GC-MS analysis. The results showed that, in comparison with the WT, the content of several long-chain fatty acids such as C15:1, C16:0, ethyl-C16, C18:1, C22:1, and C22:2 changed greatly in anthers of SiPKSA-overexpressing plants during the anther development compared with the WT (Fig. 7A). In addition, three phenolic compounds $(\mathrm{C} 14-\mathrm{OH}, \mathrm{C} 15-\mathrm{OH}$, and $\mathrm{C} 25-2 \mathrm{OH})$ and one fatty alcohol (Phytol) were increased significantly at stages 6-8 in SiPKSA-overexpressing plants (Fig. 7B). The results indicated that over-expression of SiPKSA affected fatty acid metabolism in anthers of transgenic Arabidopsis.

\section{Discussion}

\section{SiPKSA is a typical chalcone synthase with a similar function as its homologous}

Polyketide compounds are usually produced under the action of polyketide synthases (PKSs). PKSs can be divided into three types (I, II, and III) according to the protein structure $[39,40]$. Type III PKSs, also known as a chalcone synthase (CHS) superfamily, are responsible for the biosynthesis of monocyclic or bicyclic aromatics polyketides compounds [41]. These type III PKSs had a highly conserved catalytic active center (Cys164-His303-Asn336), and their active amino acid residues, generally Thr197, Phe215, Gly256, and Ser338, were located in the cavity of the active center [42]. While the active site and amino acid residues of different functions of type III PKSs are slightly different, so they will ultimately produce diverse products [43]. A series of type III PKSs with different functions have been characterized in plants, such as chalcone synthase (CHS), stilbene synthase (STS), acribone synthase (ACS), and 2-pyrone synthase (2-PS). It has been reported that plant-specific CHS-like type III PKSs LAP6/PKSA and $\angle A P 5 / P K S B$ were specifically expressed in anthers and critical for pollen development, and their mutant shows thinner exine compared with the WT [20]. OsPKS1, a homolog of LAP6/PKSA, also has a similar function. The OSPKS1 knockout mutant generated by the CRISPR/Cas9 genomic editing tool exhibited a complete male-sterile phenotype with the anthers failed to produce any viable pollen grains, and moreover, the mutant exhibited defective pollen sexine formation [16]. Consistent with it, a singlenucleotide polymorphism (SNP) mutation of the OSPKS1 gene also showed the same phenotype of male sterility caused by defective pollen wall [17]. Interference expression of NtPKSA also caused disorganized pollen surface sculptures in tobacco [22].

In our study, the phylogenic analysis results showed that SiPKSA was clustered together with PKSAs in other species and multiple alignment results showed that SiPKSA contained a typical chalcone synthase structure and possessed highly conserved catalytic active centers and active amino acid residues in consistency with AtPKSA, BnPKSA and OsPKSA/ OsPKS1 (Fig. 1). These findings indicated that SiPKSA was a type III PKSs and was homologous to other PKSAs, suggesting that SiPKSA might produce similar products and have similar functions with these PKSAs. In agreement with it, SiPKSA was specifically 
expressed in sesame flower buds, and the expression of SiPKSA was higher in sesame sterile anthers than in the fertile anthers during tetrad release and microspores development stages (Fig. 2). Besides, Overexpression of SiPKSA in Arabidopsis caused transgenic plants male sterile. The pollen grains of SiPKSA-overexpressing plants were collapsed and embedded with a defective pollen wall (Fig. 4). Further TEM observation showed that the pollen exine of SiPKSA-overexpressing plants was thicker than that of wild-type plants (Fig. 5), consistent with the phenotypes in mutants of other enzymes involved in sporopollenin biosynthesis, which lack exine or have much thinner exine layers, suggesting that SiPKSA function similar as its homologs.

\section{SiPKSA is required for proper pollen exine formation and might be involved in sporopollenin biosynthesis}

The main component of pollen exine is sporopollenin. It was noteworthy that an ancient biochemical pathway for the biosynthesis of sporopollenin had been widely proposed [7, 9, 22], and CYP704B1, MS2, TKPR1, TKPR2, PKSA/LAP6, and PKSB/LAP5, were considered as the key enzymes involved in sporopollenin biosynthesis [19, 20,44-48]. Mutants of these genes all showed defective or absent pollen exine, suggesting that down-regulation of these genes causes decreased sporopollenin biosynthesis. In our study, the pollen exine layer of SiPKSA-overexpressing plants was thicker compared with WT (Fig. 4, Fig. 5), suggesting that the sporopollenin might be abundantly accumulated on the surface of microspores of SiPKSA-overexpressing plants. This was supported by the expression analysis of sporopollenin synthesis-related genes, such as MS2, TKPR1, TKPR2, and CYP704B1, which were upregulated in SiPKSA-overexpressing plants during stage 6-8 compared with the controls (Fig. 6). Similar phenotypes were observed in male sterile line $1335 \mathrm{~A}$ of cotton, which was male sterile due to thicker nexine, and the up-regulation of the expression levels of sporopollenin synthesis-related genes caused excessive sporopollenin accumulation, finally lead to male sterility [49].

Many studies have demonstrated that sporopollenin is a complex biopolymer composed mainly of fatty acids, phenolic and aliphatic compounds [7, 14]. With the revelation of some genes related to sporopollenin synthesis, more and more biochemical experiments in vitro have been conducted to explore their biochemical function. Studies have shown that AtPKSA and the closely related AtPKSB can both catalyze the condensation of long-chain fatty acid acyl-CoA and malonyl-CoA to produce a-pyrone products. Accordingly, AtPKSA is known as a multifunctional enzyme and vital for the synthesis of both pollen exine fatty acids and phenolics $[19,20]$. Simultaneously, in rice, OsLAP6/OsPKS1 shared similar products of enzymatic reaction with AtPKSA/LAP6 [22]. Moreover, PpASCL, a homolog of AtPKSA/LAP6 in moss, was encoding a sporophyte-specific enzyme that had similar catalytic activity to AtPKSA/LAP6 in vitro [21]. In agreement with that, in our study, the contents of various long-chain fatty acids were affected in anthers of SiPKSA-overexpressing plants compared with the wild-type plants. Also, the contents of several phenolic compounds increased in the SiPKSA-overexpressing lines (Fig. 7). According to these results, we deduced that overexpression of SiPKSA might disrupt the sporopollenin biosynthesis 
in transgenic Arabidopsis, leading to excessive accumulation of sporopollenin, thicker pollen exine layer, which cause male sterile. Further experiments were needed to explore the intricate regulatory mechanism.

\section{Conclusion}

In this study, we cloned and characterized the sesame polyketide synthase A (SiPKSA). Sequence analysis and tissue expression pattern analysis showed that SiPKSA is a polyketide synthase closely related to the development of sesame anthers. SiPKSA-overexpressing Arabidopsis plants showed an obvious male-sterile phenotype. Ultrastructure observation revealed that the pollen grains of transgenic Arabidopsis were shrunken and collapsed, and the pollen exine layer was thicker than WT. Moreover, the expression levels of sporopollenin biosynthesis-related genes and fatty acids metabolism were altered in anthers of SiPKSA-overexpressing plants. Our results highlighted that overexpression of SiPKSA might cause excessive sporopollenin biosynthesis, forming thicker pollen exine layer, and then leading to male sterile, suggesting that its manipulation might improve hybrid breeding in sesame and other crop species.

\section{Materials And Methods}

\section{Plant materials and growth conditions}

The male-sterile mutant $95 \mathrm{~ms}-5 \mathrm{~A}$ and its fertile segregant 95ms-5B [29] were cultivated under natural conditions at the experimental station of the Oil Crops Research Institute of the Chinese Academy of Agricultural Sciences (OCRI-CASS, Wuhan, China). Arabidopsis ecotype Colombia (Col-0) was grown under standard growth conditions, the temperature was maintained at approximately $22^{\circ} \mathrm{C}$, the light intensity was $130 \mu \mathrm{E} / \mathrm{m}^{2} \mathrm{~s}$, and the photoperiod was $16 \mathrm{~h}$ light/ $8 \mathrm{~h}$ dark. All the plant materials were provided by the OCRI-CASS.

\section{Clone and sequence analysis}

The ORF of SiPKSA was amplified with the specific gene primer SiPKSA-f (5'ATGTCCAACATCATCATCAACAGC-3') and SiPKSA-r (5'-TCAAAGACTCCTAAGAAGAATGCCT-3') via PCR using the fertile sesame anthers at the tetrad stage as the template. The physical and chemical properties of SiPKSA were analyzed using the EXPASY database (https://web.expasy.org/protparam/). The PLANTCARE and PLACE databases were used to analyze the cis-acting elements of SiPKSA [31, 32].

For multiple alignment analysis, SiPKSA and three other PKSAs (AtPKSA, At1g02050; OsPKSA, NP_001064891; BnPKSA, XP_013701181) were aligned by the ClustalX program. For phylogenetic analysis, SiPKSA and selected PKSs from other species (Arabidopsis thaliana, At4g34850; Brassica napus, XP_013707052; Helianthus annuus, XP_022010182; Glycine max, XP_003537759, XP_003516799; Populus trichocarpa, XP_002302511; Oryza sativa, XP_015646301; Zea mays, PWZ54429; Arachis hypogaea, XP_015971059, XP_015952719; Mesicago truncatula, XP_024638419; Brassica rapa, XP_009108697) were subsequently constructed via MEGA-X with the neighbor-joining method as the default and 1000 bootstrap replicates [33]. All sequences were downloaded from the NCBI database. 


\section{Vector construction and plant transformation}

First, the ORF of SiPKSA was inserted into TSV-007S vector (Tsingke, China) and sequenced. Subsequently, through double enzyme digestion (restriction site: Nde\and Sma囚), the fragment was ligated into PRI101-AN (Takara, Japan) to generate the overexpression vector (35S::SiPKSA) according to previously described methods $[34,35]$. The expression vector was then introduced into Arabidopsis thaliana (Col-0) through the Agrobacterium tumefaciens (strain GV3101). Arabidopsis plants transformed with empty vector (EV) and wild-type Arabidopsis (WT) were used as the control.

\section{Plant morphology and pollen viability analysis}

When the seedlings were eight weeks old, the phenotypes of transgenic plants were photographed using a Nikon D90 digital camera. Pollen grains from wild type plants transformed with empty vector and SiPKSA-overexpressing plants were stained with $0.5 \%$ acetyl carmine reagent, and the photographs were captured using an optical microscope (IX71, Olympus). All plump and darkly stained pollen grains were determined as fertile, and the collapsed and unstained pollen grains were considered sterile.

\section{Ultrastructure observation}

For Scanning electron microscope (SEM) observation, pollen grains sampled from freshly dehisced anthers of WT, EV, and transgenic lines were dehydrated in a graded ethanol series. The dehydrated samples were dried to the critical point in liquid $\mathrm{CO}_{2}$ and coated with gold particles. The images were acquired by a scanning electron microscope (VEGA3, Tescan).

For Transmission electron microscope (TEM) observation, anthers at different developmental stages collected from WT and SiPKSA-overexpressing plants were fixed in $2.5 \%$ glutaraldehyde at $4^{\circ} \mathrm{C}$ overnight. Next, the samples were post-fixed with $1 \%$ osmium tetroxide, and subsequently dehydrated twice in $30 \%$, $50 \%, 70 \%, 80 \%, 90 \%, 95 \%$, and $100 \%$ ethanol for 15-20 minutes each time. After that, the samples were infiltrated with a mixture of acetone and epoxy resin in different proportions. The dehydrated samples were then embedded in epoxy resin and polymerized at $60^{\circ} \mathrm{C}$ (incubator) for 48 hours. Further, they were sliced into the thickness of 80-100nm by an ultra-thin slicer (EMUC7, Leica), and stained with the uranium-lead double staining solution. The pictures were captured using the transmission electron microscope (TecnaiG ${ }^{2} 20$ TWIN). The length of the baculum was measured using the Nano measurer 1.2 software (http://emuch.net/html/201402/7022970.html). Three biological replicates were performed, and three microspores were measured for each replicate. The measurements were repeated five times for each sample.

\section{RNA isolation, RT-PCR and qRT-PCR analysis}

Sesame root, stem, leaf, flower buds, capsules were collected for expression profile analysis of SiPKSA in sesame. To compare the expression difference of SiPKSA between sterile sesame plants (95ms-5A) and the fertile plants (95ms-5B), anthers at different developmental stages (tetrad stage, microspore development stage, and mature pollen stage) from sterile sesame plants (95ms-5A) and the fertile plants 
(95ms-5B) were respectively sampled. Expression was normalized to SiActin (SIN_1009011) [36]. For expression analysis of sporopollenin biosynthesis-related genes in Arabidopsis anthers at different development stages (stage 6-8, 9-10,11-12,13-14) from wild type, plants transformed with empty vector and SiPKSA-overexpressing plants were sampled. Expression was normalized to AtActin (At3g18780) [19]. Total RNA was respectively extracted from each sample using the Trizol reagent (Invitrogen, USA). The first-strand cDNA was synthesized by the SuperScript II reverse transcriptase (Vazyme Biotech, Nanjing, China). qRT-PCR was carried out using the Roche Light Cycler 480 system. The relative expression level was calculated using the $2^{-\triangle \mathrm{Ct}}$ method [37]. The primers used in this study were listed in Table S1.

\section{Fatty acid and its derivates measurement}

Anthers at different developmental stages sampled from WT and the SiPKSA-overexpressing plants were respectively extracted twice with $1 \mathrm{~mL}$ of chloroform for 2 hours. Next, the mixed supernatant was reextracted and dried with a nitrogen blower. Finally, $0.25 \mathrm{~mL}$ of $5 \%$ potassium hydroxide-methanol solution was added, and the mixture was incubated at $60^{\circ} \mathrm{C}$ for 30 minutes and subsequently quenched with $\mathrm{n}$ hexane. The final supernatant was analyzed by Gas Chromatography-Mass Spectrometry (GC-MS). The chromatographic conditions by GC-MS were as follows: inlet temperature $280^{\circ} \mathrm{C}$, split ratio $20: 1$, carrier gas: He, carrier gas flow rate: $1.0 \mathrm{ml} / \mathrm{min}$, ion source temperature $230^{\circ} \mathrm{C}$, scanning mass range $35 \sim 800_{\mathrm{M}}$ The injection volume was $1 \mu \mathrm{L}$. The temperature rising program: The initial temperature was $50^{\circ} \mathrm{C}$ and kept for 1 minute; the temperature was raised to $200^{\circ} \mathrm{C}$ gradually $\left(5^{\circ} \mathrm{C} / \mathrm{min}\right)$; finally, the temperature was raised to $230^{\circ} \mathrm{C}$ and kept for 10 minutes.

\section{Statistical analysis}

All the experiments were performed by three biological replicates, and the presented values are mean \pm SD. Statistical significance was determined using Student's t-test $\left({ }^{*} P<0.05,{ }^{*} P<0.01\right)$.

\section{Abbreviations}

ORF: Open Reading Frame

WT:Wild Type

EV: Transformed Plants with Empty Vector

OX: Overexpression Lines

RT-PCR: Reverse Transcription-Polymerase Chain Reaction

qRT-PCR: Quantitative Real-Time PCR

SEM: Scanning Electron Microscope 
TEM: Transmission Electron Microscope

\section{Declarations}

\section{Acknowledgments}

Not application.

\section{Author's Contributions}

$T Y L, T Z$, and $Y Z Z$ conceived and designed the project and experiments. TYL and YXY performed the experiments. TYL analyzed the data and wrote the manuscript. HYL and FZ assisted in preparing the materials. YZZ, TZ, and DSSK revised the manuscript. All authors read and approved the manuscript.

\section{Funding}

This research was funded by the National Natural Science Foundation of China (No. 31701468, No. 31771877), China Agriculture Research System of MOF and MARA (CARS-14), and the Agricultural Science and Technology Innovation Project of the Chinese Academy of Agricultural Sciences (CAASASTIP-2013-OCRI).

\section{Availability of data and materials}

All data generated or analyzed during this study are included in this published article and its supplementary information files. The datasets used and/or analyzed during the current study are available from the corresponding author on reasonable request.

\section{Ethics approval and consent to participate}

Not applicable.

\section{Consent for publication}

Not applicable.

\section{Competing interests}

The authors declare that they have no competing interests. 


\section{Author details}

${ }^{1}$ Key Laboratory of Biology and Genetic Improvement of Oil Crops, Ministry of Agriculture of the People's Republic of China, Oil Crops Research Institute, Chinese Academy of Agricultural Sciences, Wuhan 430062, China.

\section{References}

1. Orruno E, Morgan MRA: Purification and characterisation of the $7 \mathrm{~S}$ globulin storage protein from sesame (Sesamum indicum L.). Food Chem. 2007, 100(3):926-934.

2. Anilakumar KR, Pal A, Khanum F, Bawa AS: Nutritional, medicinal and industrial uses of sesame (Sesamum indicum L.) seeds - an overview. Agriculturae Conspectus Scientificus (Poljoprivredna Znanstvena Smotra). 2010, 75(4):159-168.

3. Majdalawieh AF, Dalibalta S, Yousef SM: Effects of sesamin on fatty acid and cholesterol metabolism, macrophage cholesterol homeostasis and serum lipid profile: A comprehensive review. European Journal of Pharmacology. 2020, 885:173417.

4. Majdalawieh AF, Mansour ZR: Sesamol, a major lignan in sesame seeds (Sesamum indicum): Anticancer properties and mechanisms of action. European Journal of Pharmacology. 2019, 855:75-89.

5. Majdalawieh AF, Massri M, Nasrallah GK: A comprehensive review on the anti-cancer properties and mechanisms of action of sesamin, a lignan in sesame seeds (Sesamum indicum). European Journal of Pharmacology. 2017, 815:512-521.

6. Wu M-S, Aquino LBB, Barbaza MYU, Hsieh C-L, De Castro-Cruz KA, Yang L-L, Tsai P-W: Antiinflammatory and anticancer properties of bioactive compounds from Sesamum indicum L.-A Review. Molecules. 2019, 24(24):4424.

7. Ariizumi T, Toriyama K: Genetic regulation of sporopollenin synthesis and pollen exine development. Annual Review of Plant Biology. 2011, 62(1):437-460.

8. Sanders BAQ, Weterings K, et al. : Anther developmental defects in Arabidopsis thaliana. Sexual Plant Reproduction. 1999, 11(6):297-322.

9. Gomez JF, Talle B, Wilson ZA: Anther and pollen development: A conserved developmental pathway. Journal of Integrative Plant Biology. 2015, 57(11):876-891.

10. Scott RJ, Spielman M, Dickinson HG: Stamen structure and function. Plant Cell. 2004, 16:S46-S60.

11. Zinkl GM, Zwiebel BI, Grier DG, Preuss D: Pollen-stigma adhesion in Arabidopsis: a species-specific interaction mediated by lipophilic molecules in the pollen exine. Development. 1999, 126(23):54315440.

12. Blackmore S, Wortley AH, Skvarla JJ, Rowley JR: Pollen wall development in flowering plants. New Phytologist. 2007, 174(3):483-498.

13. Liu L, Fan X-d: Tapetum: regulation and role in sporopollenin biosynthesis in Arabidopsis. Plant Molecular Biology. 2013, 83(3):165-175. 
14. Shi J, Cui M, Yang L, Kim Y-J, Zhang D: Genetic and biochemical mechanisms of pollen wall development. Trends in Plant Science. 2015, 20(11):741-753.

15. Quilichini TD, Grienenberger E, Douglas CJ: The biosynthesis, composition and assembly of the outer pollen wall: A tough case to crack. Phytochemistry. 2015, 113:170-182.

16. Shi Q-S, Wang K-Q, Li Y-L, Zhou L, Xiong S-X, Han Y, Zhang Y-F, Yang N-Y, Yang Z-N, Zhu J: OsPKS1 is required for sexine layer formation, which shows functional conservation between rice and Arabidopsis. Plant Science. 2018, 277:145-154.

17. Zou T, Xiao Q, Li W, Luo T, Yuan G, He Z, Liu M, Li Q, Xu P, Zhu J et al: OsLAP6/OsPKS1, an orthologue of Arabidopsis PKSA/LAP6, is critical for proper pollen exine formation. Rice. 2017, 10(1):53.

18. Zhang D, Shi J, Yang X: Role of lipid metabolism in plant pollen exine development. Sub-cellular biochemistry. 2016, 86.

19. Kim SS, Grienenberger E, Lallemand B, Colpitts CC, Kim SY, Souza CdA, Geoffroy P, Heintz D, Krahn D, Kaiser $M$ et al: LAP6/POLYKETIDE SYNTHASE A and LAP5/POLYKETIDE SYNTHASE B Encode Hydroxyalkyl a-Pyrone Synthases Required for Pollen Development and Sporopollenin Biosynthesis in Arabidopsis thaliana. The Plant Cell. 2010, 22(12):4045-4066.

20. Dobritsa AA, Lei Z, Nishikawa S-i, Urbanczyk-Wochniak E, Huhman DV, Preuss D, Sumner LW: LAP5 and LAP6 encode anther-specific proteins with similarity to chalcone synthase essential for pollen exine development in Arabidopsis. Plant Physiology. 2010, 153(3):937-955.

21. Colpitts CC, Kim SS, Posehn SE, Jepson C, Kim SY, Wiedemann G, Reski R, Wee AGH, Douglas CJ, Suh D-Y: PpASCL, a moss ortholog of anther-specific chalcone synthase-like enzymes, is a hydroxyalkylpyrone synthase involved in an evolutionarily conserved sporopollenin biosynthesis pathway. New Phytologist. 2011, 192(4):855-868.

22. Wang Y, Lin Y-C, So J, Du Y, Lo C: Conserved metabolic steps for sporopollenin precursor formation in tobacco and rice. Physiologia Plantarum. 2013, 149(1):13-24.

23. Qin M, Tian T, Xia S, Wang Z, Song L, Yi B, Wen J, Shen J, Ma C, Fu T et al: Heterodimer formation of BnPKSA or BnPKSB with BnACOS5 constitutes a multienzyme complex in tapetal cells and is involved in male reproductive development in Brassica napus. Plant and Cell Physiology. 2016, 57(8):1643-1656.

24. Pal BP: Studies in hybrid vigour. I. Notes on the manifestation of hybrid vigour in gram, Sesamum, chilli and maize. Indian Journal of Genetics and Plant Breeding. 1945, 5:106-121.

25. Ding FY, Jiang JP, Zhang DX, Li GS: A study on relationship between heterosis and effects of combining ability in sesame. Acta Agriculturae Boreali-Sinica. 1991, 6(3):44-46.

26. Murty DS: Heterosis, combining ability and reciprocal effects for agronomic and chemical characters in sesame. Theoretical and Applied Genetics. 1975, 45(7):294-299.

27. Tu L, Liang X, Wang W, Zheng Y, Liu J: Studies on genetic male sterility in sesame (Sesamum indicum L.). Acta Agriculturae Boreali-Sinica. 1995, 10(1):34-39. 
28. Wang Q, Wenxin CAO, Guizhen XU: Study on the recessive-male sterile lines(0176A,54 - 8 A)and their utilization in sesame breeding. Chinese Journal of Oil Crop Sciences. 2007, 29(2):157-161.

29. Zhao YZ, Yang MM, Wu K, Liu HY, Wu JS, Liu KD: Characterization and genetic mapping of a novel recessive genic male sterile gene in sesame (Sesamum indicum L.). Mol Breeding. 2013, 32(4):901908.

30. Zhou T, Hao G, Yang Y, Liu H, Yang M, Zhao Y: Sicwinv1, a cell wall invertase from sesame, is involved in anther development. Journal of Plant Growth Regulation. 2019, 38(4):1274-1286.

31. Lescot M, Dehais P, Thijs G, Marchal K, Moreau Y, Van de Peer Y, Rouze P, Rombauts S: PlantCARE, a database of plant cis-acting regulatory elements and a portal to tools for in silico analysis of promoter sequences. Nucleic Acids Research. 2002, 30(1):325-327.

32. Higo $\mathrm{K}$, Ugawa $\mathrm{Y}$, Iwamoto $\mathrm{M}$, Korenaga T: Plant cis-acting regulatory DNA elements (PLACE) database: 1999. Nucleic Acids Research. 1999, 27(1):297-300.

33. Kumar S, Stecher G, Li M, Knyaz C, Tamura K: MEGA X: molecular evolutionary genetics analysis across computing platforms. Molecular Biology and Evolution. 2018, 35(6):1547-1549.

34. Yang X Y, Li J G, Pei M, Gu H, Chen Z L, Qu L J. Over-expression of a flower-specific transcription factor gene AtMYB24 causes aberrant anther development. Plant Cell Reports. 2007, 26(2):219-228.

35. Wang Y, Li Y, He S P, Gao Y, Wang N N, Lu R, Li X B. A cotton (Gossypium hirsutum) WRKY transcription factor (GhWRKY22) participates in regulating anther/pollen development. Plant Physiology and Biochemistry. 2019, 141:231-239.

36. Li D, Liu P, Yu J, Wang L, Dossa K, Zhang Y, Zhou R, Wei X, Zhang X. Genome-wide analysis of WRKY gene family in the sesame genome and identification of the WRKY genes involved in responses to abiotic stresses. BMC Plant Biology. 2017, 17:152.

37. Schmittgen TD, Livak KJ: Analyzing real-time PCR data by the comparative C-T method. Nature Protocols. 2008, 3(6):1101-1108.

38. Ahlers F, Bubert H, Steuernagel S, Wiermann R: The nature of oxygen in sporopollenin from the pollen of Typha angustifolia L. Zeitschrift Fur Naturforschung C-a Journal of Biosciences. 2000, 55(34):129-136.

39. Jez JM, Bowman ME, Dixon RA, Noel JP: Structure and mechanism of the evolutionarily unique plant enzyme chalcone isomerase. Nature Structural Biology. 2000, 7(9):786-791.

40. Hopwood DA: Genetic contributions to understanding polyketide synthases. Chemical Reviews. 1997, 97(7):2465-2497.

41. Shen B, Cheng Y-Q, Christenson SD, Jiangi H, Ju J, Kwon H-J, Lim S-K, Liu W, Nonaka K, Seo J-W et al: Polyketide biosynthesis beyond the type I, II, and III polyketide synthase paradigms: a progress report. In: Polyketides: Biosynthesis, Biological Activity, and Genetic Engineering. Edited by Rimando AM, Baerson SR, vol. 955; 2007: 154-166.

42. Abe I, Utsumi Y, Oguro S, Morita H, Sano Y, Noguchi H: A plant type III polyketide synthase that produces pentaketide chromone. Journal of the American Chemical Society. 2005, 127(5):13621363. 
43. Austin MB, Bowman ME, Ferrer JL, Schroder J, Noel JP: An aldol switch discovered in stilbene synthases mediates cyclization specificity of type III polyketide synthases. Chemistry \& Biology. 2004, 11(9):1179-1194.

44. Dobritsa AA, Geanconteri A, Shrestha J, Carlson A, Kooyers N, Coerper D, Urbanczyk-Wochniak E, Bench BJ, Sumner LW, Swanson R et al: A Large-scale genetic screen in Arabidopsis to identify genes involved in pollen exine production. Plant Physiology. 2011, 157(2):947-970.

45. Dobritsa AA, Shrestha J, Morant M, Pinot F, Matsuno M, Swanson R, Møller BL, Preuss D: CYP704B1 is a long-chain fatty acid $\omega$-Hydroxylase essential for sporopollenin synthesis in pollen of Arabidopsis. Plant Physiology. 2009, 151(2):574-589.

46. Grienenberger E, Kim SS, Lallemand B, Geoffroy P, Heintz D, Souza CdA, Heitz T, Douglas CJ, Legrand M: Analysis of TETRAKETIDE a-PYRONE REDUCTASE function in Arabidopsis thaliana reveals a previously unknown, but conserved, biochemical pathway in sporopollenin monomer biosynthesis. The Plant Cell. 2010, 22(12):4067-4083.

47. de Azevedo Souza C, Kim SS, Koch S, Kienow L, Schneider K, McKim SM, Haughn GW, Kombrink E, Douglas CJ: A novel fatty acyl-COA synthetase is required for pollen development and sporopollenin biosynthesis in Arabidopsis. The Plant Cell. 2009, 21(2):507-525.

48. Lallemand B, Erhardt M, Heitz T, Legrand M: Sporopollenin biosynthetic enzymes interact and constitute a metabolon localized to the endoplasmic reticulum of tapetum cells. Plant Physiology. 2013, 162(2):616-625.

49. Wu Y, Ling M, Wu Z, Li Y, Zhu L, Yang X, Yuan D. Defective pollen wall contributes to male sterility in the male sterile line 1355A of cotton. Scientific Reports. 2015, 5: 9608.

\section{Figures}


A Chal_sti synt $\mathrm{N}$
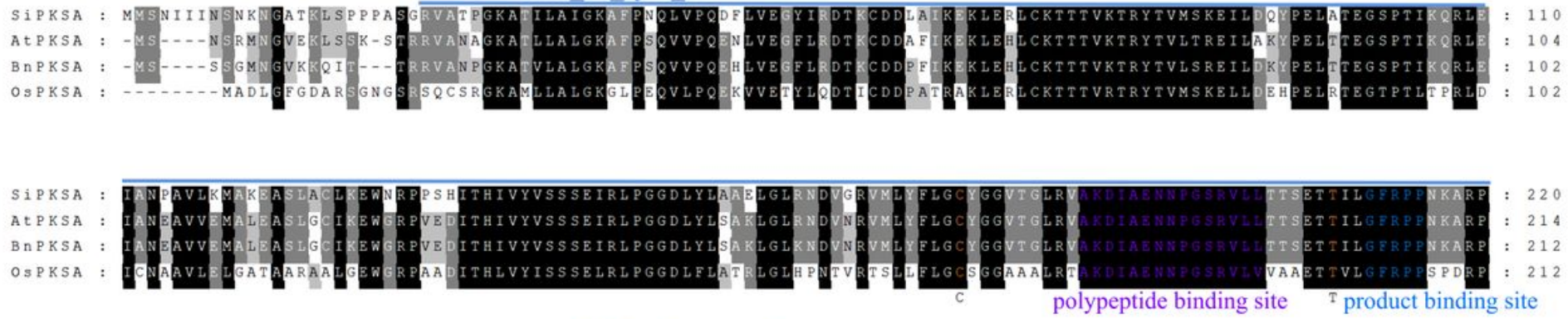

Chal sti synt $\mathrm{C}$

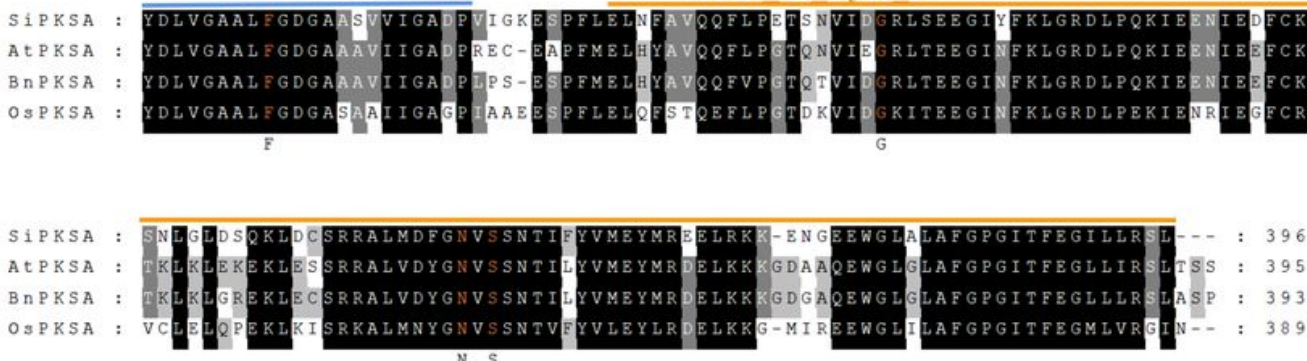

$\mathrm{B}$

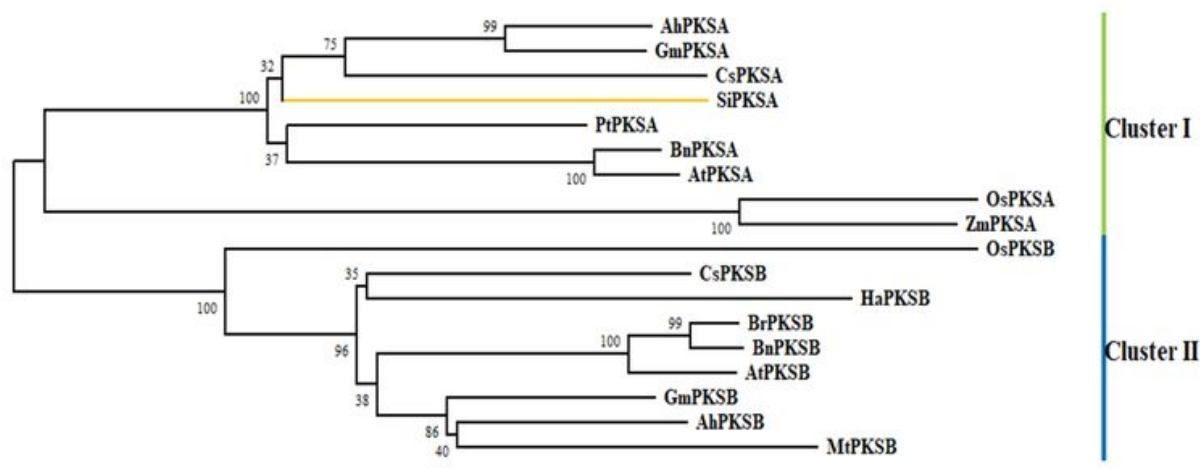

\section{Figure 1}

Multiple sequence alignment and phylogenic analysis of SiPKSA with the plant-specific type III polyketide synthase (PKSs) in other species. (A) Protein sequence alignment of SiPKSA with anther developmentalrelated PKSA from other crops. The conservative sites are underlined and amino acid residues are framed. AtPKSA (At1g02050), BnPKSA (XP_013701181.1), OsPKSA (NP_001064891.1). (B) Phylogenetic analysis of SiPKSA with PKSs from other species. Sesame indium (Si); Arabidopsis thaliana (At); Zea mays (Zm); Glycine max (Gm); Populus trichocarpa (Pt); Oryza sativa (Os); Brassica napus (Bn); Medicago truncatula (Mt); Citrus sinensis (Cs); Helianthus annuus (Ha); Arachis hypogaea (Ah); Brassica rapa $(\mathrm{Br})$. 


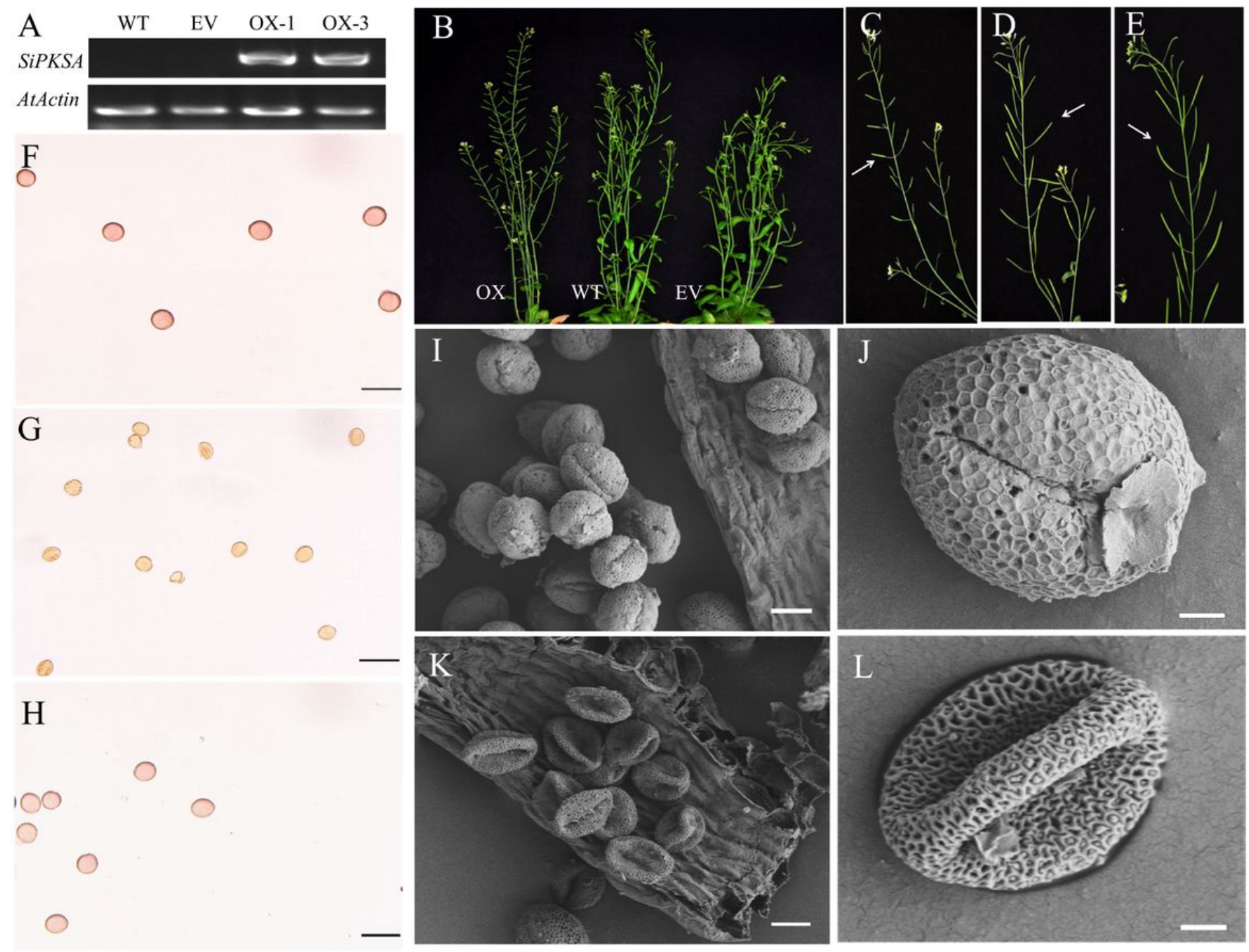

Figure 2

Expression analysis of SiPKSA in sesame. (A-B) The expression profile of SiPKSA in different sesame tissues by RT-PCR (A) and qRT-PCR analysis (B). (C-D) RT-PCR (C) and qRT-PCR analysis (D) of the expression of SiPKSA in sesame fertile and sterile anthers at different developmental stages. Si-Actin (SIN_1009011) was used as the internal reference control. 
A

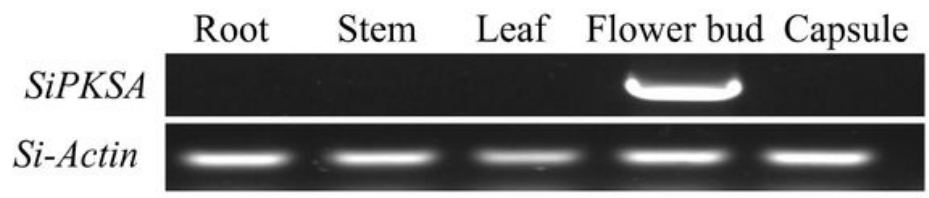

B

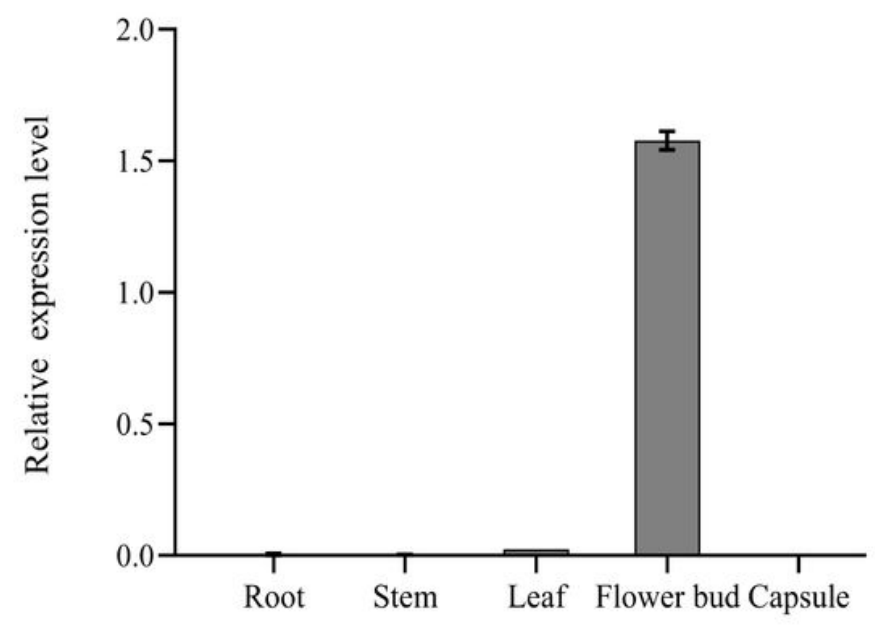

C
SiPKSA

Si-Actin
F-t $\quad$ F-md F-mp $\quad$ S-t $\quad$ S-md $\quad$ S-mp

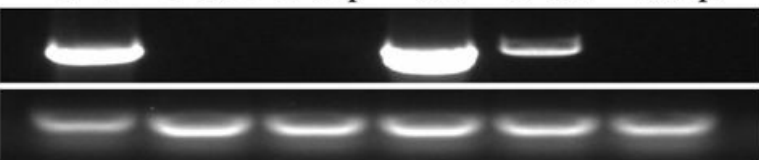

D

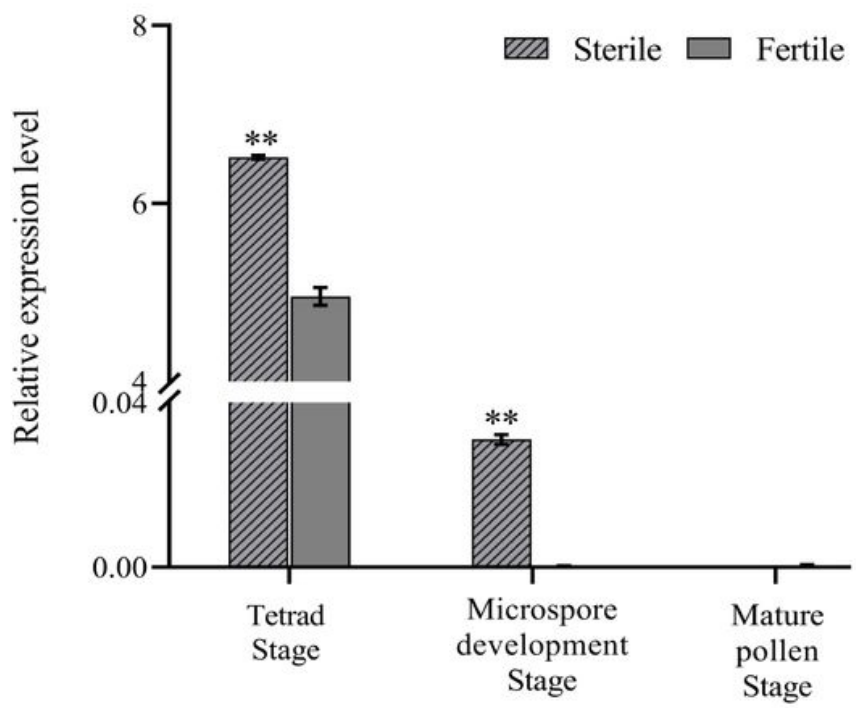

Figure 3

Ectopic expression of SiPKSA in Arabidopsis caused transgenic plants male sterile. (A) RT-PCR analysis of SiPKSA in SiPKSA-overexpressing lines (OX-1, OX-2, OX-3), wild-type (WT) and plants transformed with empty vector (EV). At-Actin (At3g18780) was used as an internal standard. (B) Phenotype of 8-weeks-old SiPKSA-overexpressing Arabidopsis plants. (C-E) Magnified image of SiPKSA-overexpressing plant (C), WT (D), and EV (E). White arrow indicated the siliques of plants. $(F-H)$ Detection of pollen vigor of WT (F), SiPKSA-overexpressing plant $(\mathrm{G})$ and $E V(\mathrm{H})$ with $0.5 \%$ aceto carmine reagent. $(\mathrm{l}, \mathrm{J})$ Pollen grains of WT observed by SEM. (K, L) Pollen grains of SiPKSA-overexpressing plant observed by SEM. Scale bars represent $10 \mu \mathrm{m}(\mathrm{I}, \mathrm{K}), 2 \mu \mathrm{m}(\mathrm{J}, \mathrm{L})$, respectively. 


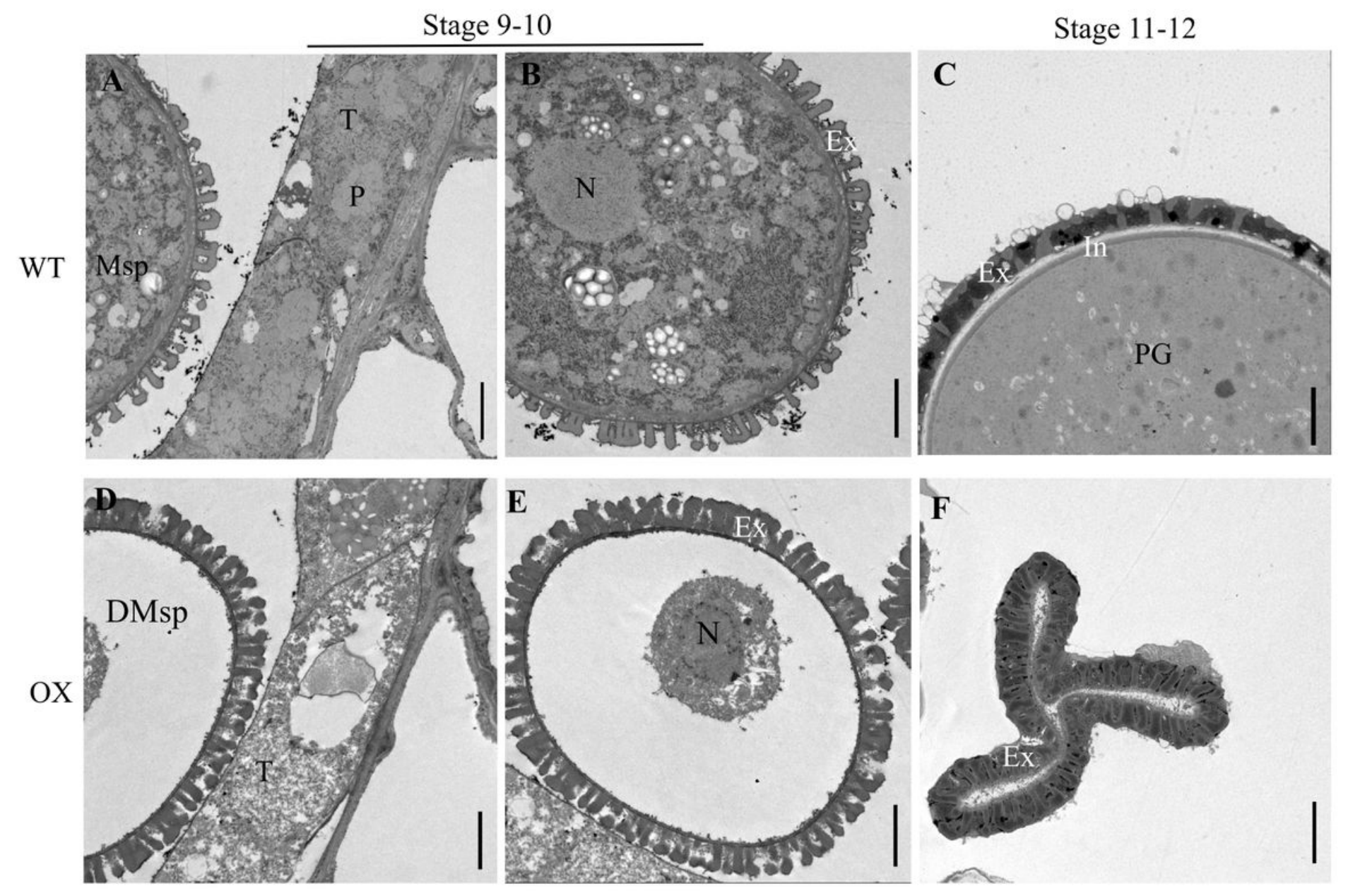

\section{Figure 4}

Transmission electron micrographs (TEM) observation of the ultrastructure of microspore and tapetum development of wild-type and SiPKSA-overexpressing plants at different anther developmental stages. (A, D) Tapetal cells of the WT (A) and SiPKSA-overexpressing plant during stage 9-10 (D). (B, E) Microspores of the WT (B) and SiPKSA-overexpressing plant during stage 9-10 (E). (C, F) Pollen wall of WT (C) and SiPKSA-overexpressing plant (F) at stage 11-12. Msp, microspore; Msp, microspore; DMsp, degenerated microspore; T, tapetum; In, intine; Ex, exine; N, nucleus; P, plastid filled with plastoglobuli; Try, tryphine; PG, pollen grain. Scale bars were $2 \mu \mathrm{m}$. 

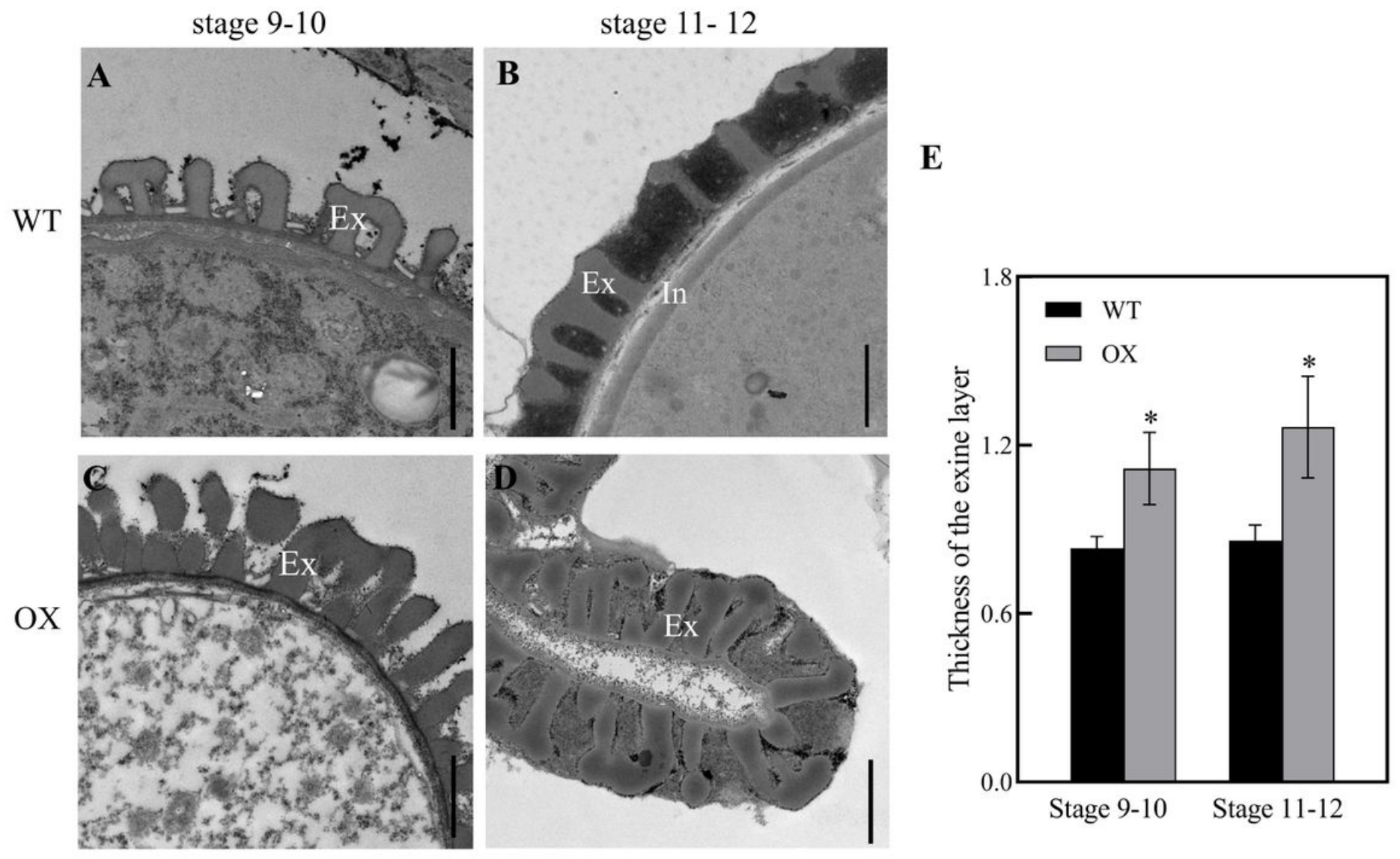

Figure 5

TEM observation of pollen wall from wild-type (WT) and SiPKSA-overexpressing plants at different anther developmental stages. (A-D) The ultrastructure of pollen wall of WT and SiPKSA-overexpressing plants at anther developmental stages 9-10 and 11-12. (E) The thickness of exine layer at stages 9-10 and 11-12. Ex, exine; In, intine. Scale bars were $1 \mu \mathrm{m}$. Three biological replicates were conducted and the value are mean \pm SD. Statistically significant differences were assessed using student's t-test $\left({ }^{*} p<0.05,{ }^{*} p<0.01\right)$. 

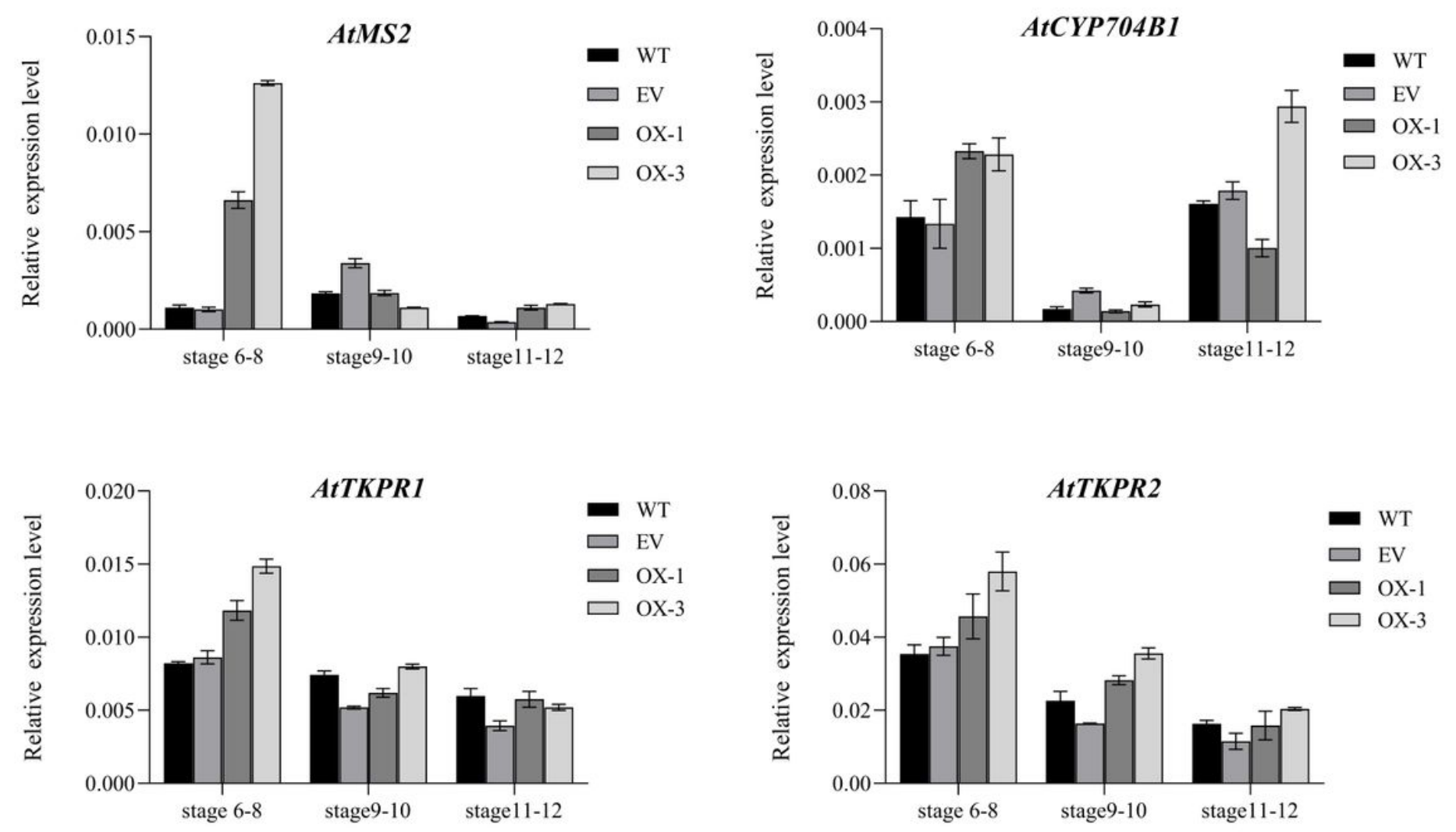

Figure 6

qRT-PCR analysis of the pollen development-related genes in the controls and SiPKSA-overexpressing plants. Three biological replicates were performed and expression was normalized to AtActin (At3g18780). Statistically significant differences were assessed using student's t-test $\left({ }^{\star} p<0.05,{ }^{*} p<0.01\right)$. AtMS2 (At3g11980), AtCYP704B1 (At1g69500), AtTKPR1 (At4g35420), AtTKPR2 (At1g62940) 
A

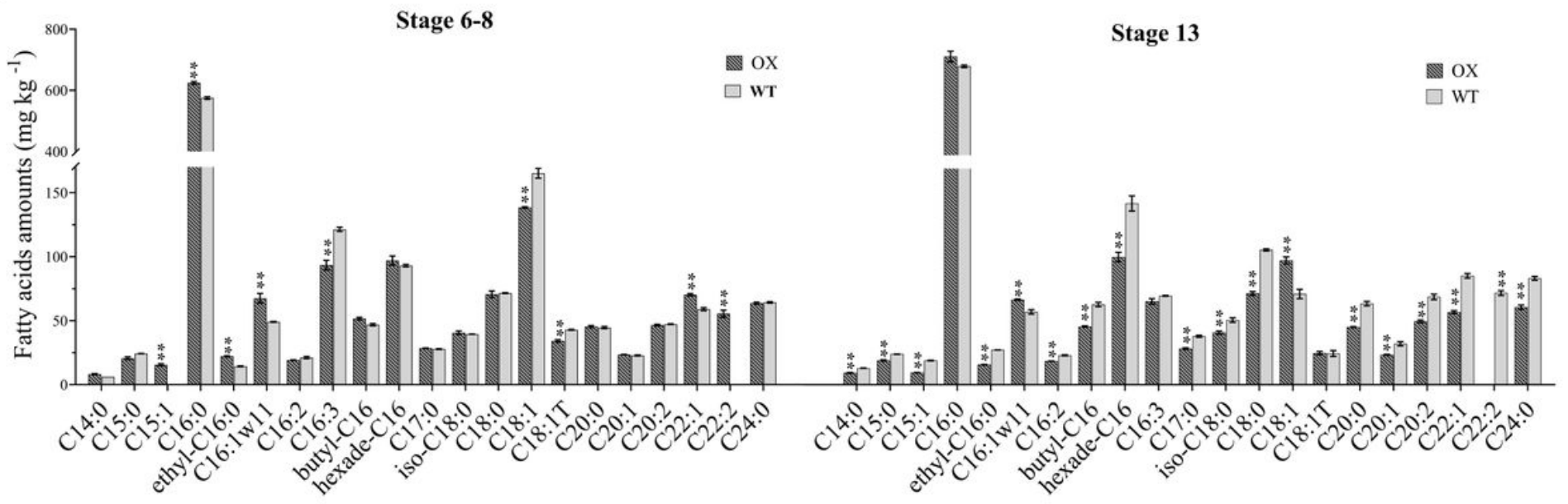

B

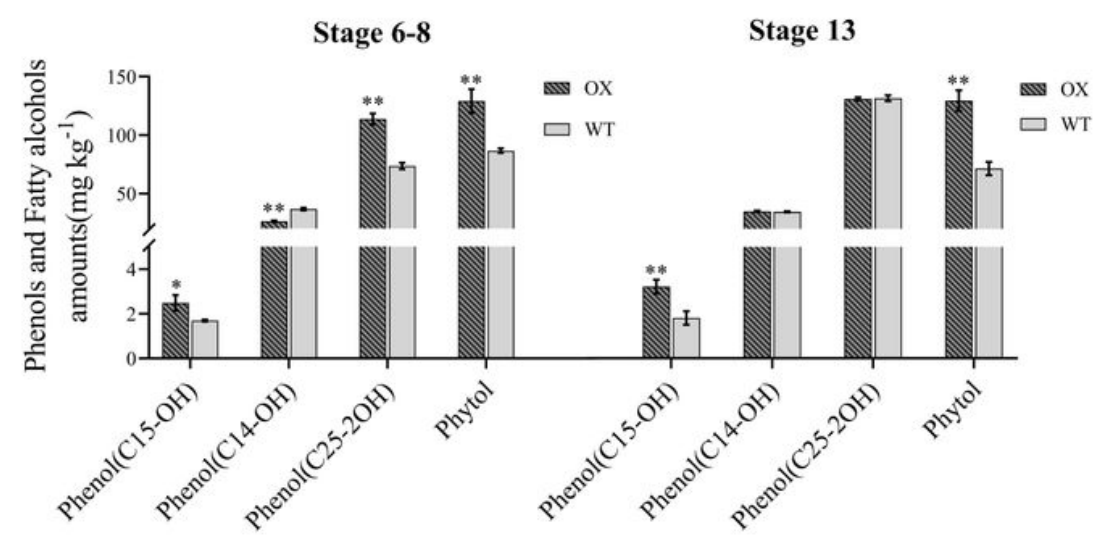

Figure 7

Measurement of fatty acids and their derivates in anthers of SiPKSA-overexpressing plant and wild type by GC-MS. (A) The content of fatty acids in anthers at stages 6-8 and 13. (B) The content of Phenols and fatty alcohol in anthers at stages 6-8 and 13. C12:0, Lauric acid methyl ester; C14:0, Methyl tetradecanoate; C15:0, Pentadecanoic acid methyl ester; C15:1, 9-Octadecenoic acid methyl ester; C16:0, Hexadecanoic acid methyl ester; ethyl-C16:0, 9-Hexadecenoic acid methyl ester; C16:1w11, 11Hexadecenoic acid methyl ester; C16:2, 7,10-Hexadecadienoic acid methyl ester; C16:3, 7,10,13Hexadecatrienoic acid methyl ester; hexade-C16, Hexadecanedioic acid dimethyl ester; butyl-C16, butyl isobutyl phthalate; C17:0, Heptadecanoic acid methyl ester; C18:0, Methyl stearate; iso-C18:0, Methyl isostearate; C18:1, (Z)-9-Octadecenoic acid methyl ester; C18:1T, 9,12,15-Octadecatrienoic acid ethyl ester; C20:0, Arachidic acid methyl ester; C20:1, cis-11-Eicosenoic acid methyl ester; C20:2, 11,13-Eicosadienoic acid methyl ester; C22:1, 13-Docosenoic acid methyl ester; C22:2, cis-13,16-Docasadienoic acid methyl ester; C24:0, Tetracosanoic acid methyl ester; Phenol(C15-OH), Butylated Hydroxytoluene; Phenol(C14$\mathrm{OH})$, 2,4-bis(1,1-dimethylethyl)-Phenol; Phenol(C25-2OH), 2,2'-methylenebis[6-(1,1-dimethylethyl)]-4methyl-Phenol.

\section{Supplementary Files}


This is a list of supplementary files associated with this preprint. Click to download.

- Supplementaryinformation.docx 